\title{
Modelling contact oxygenation and adhesive wear extension in axisymmetric flat circular fretting interfaces
}

\author{
Soha Baydoun ${ }^{a_{*}}$, Pierre Arnaud ${ }^{b_{*}}$, Siegfried Fouvry ${ }^{a_{*}}$
}

\begin{abstract}
${ }^{a}$ Ecole Centrale de Lyon, LTDS Laboratory, 36 av Guy de Collongue, 69130 Ecully, France
${ }^{\mathrm{b} C e n t r a l e S u p e l e c, ~ M S S M A T ~ L a b o r a t o r y, ~ 8-10 ~ r u e ~ J o l i o t ~ C u r i e, ~} 91190$ Gif-sur-Yvette, France

*Corresponding authors email addresses: soha.baydoun@ec-lyon.fr (S. Baydoun), pierre.arnaud@centralesupelec.fr (P. Arnaud) and siegfried.fouvry@ec-lyon.fr (S. Fouvry)
\end{abstract}

\begin{abstract}
In this study, adhesive wear extension in axisymmetric fretting contacts is modeled by applying the Contact Oxygenation Concept $(\mathrm{COC})$ which illustrates the influence of fretting solicitations on oxygen transport within the interface. Di-oxygen flow is modeled using an Advection-Dispersion-Reaction (ADR) approach which was formerly proposed assuming debris bed as a porous medium traversed by atmospheric gases. It was shown that ADR approach is not only predictive in square contacts but also in circular flat interfaces where it is capable of simulating the partition of adhesion and abrasion for a wide range of sliding frequencies and contact pressures. Moreover, a simplified analytical solution is proposed for ADR equation which is proven to be highly correlated with the numerical and experimental results.
\end{abstract}

Key words: Abrasive-adhesive fretting wear; Axisymmetric-circular flat contact; Contact oxygenation concept; Advection-Dispersion-Reaction model (ADR). 


\section{Introduction}

Micro-displacement oscillatory motion, referred to as fretting $[1,2]$, is encountered in a wide range of industrial assemblies submitted to vibrations (bridge cables [3], seismic friction dampers [4], turbo-jet engines [5], railway axels [6], etc.). It leads to a serious surface damage that impairs the performance of mechanical systems incurring industries exhaustive maintenance and renovation costs. Hence, understanding fretting wear is inevitable for saving economic and natural resources.

When relatively high displacement amplitudes are applied, gross slip fretting regime dominates leading to wear damage characterized by creation and expulsion of debris particles. Several wear mechanisms are thought to be the origin of debris particles and eventually material loss including fatigue, tribo-oxidation, abrasion, and adhesion [7]. Adhesive wear is one of the most severe forms of wear as it leads to welding of materials which might ultimately block the sliding of mechanical system inducing seizure and galling phenomena.

The transition from abrasive to adhesive wear in gross-slip fretting was shown to be dependent on different loading conditions [8-13]. This dependency can be explained by the "Contact Oxygenation Concept (COC)" [11] which suggests that adhesion appears in the contact center if the di-oxygen partial pressure $\left(P_{O_{2}}\right)$ is below a threshold value $\left(P_{O_{2}, t h}\right)$ (Fig. 1a). In the lateral sides, where di-oxygen molecules are sufficient, oxidation and abrasion prevail. Based on this concept, an "oxygen distance $d_{O}$ " parameter was defined as the distance from the contact border to the boundaries of adhesion zone after which oxygen transport becomes restricted inducing in turn seizure (Fig. 1). 


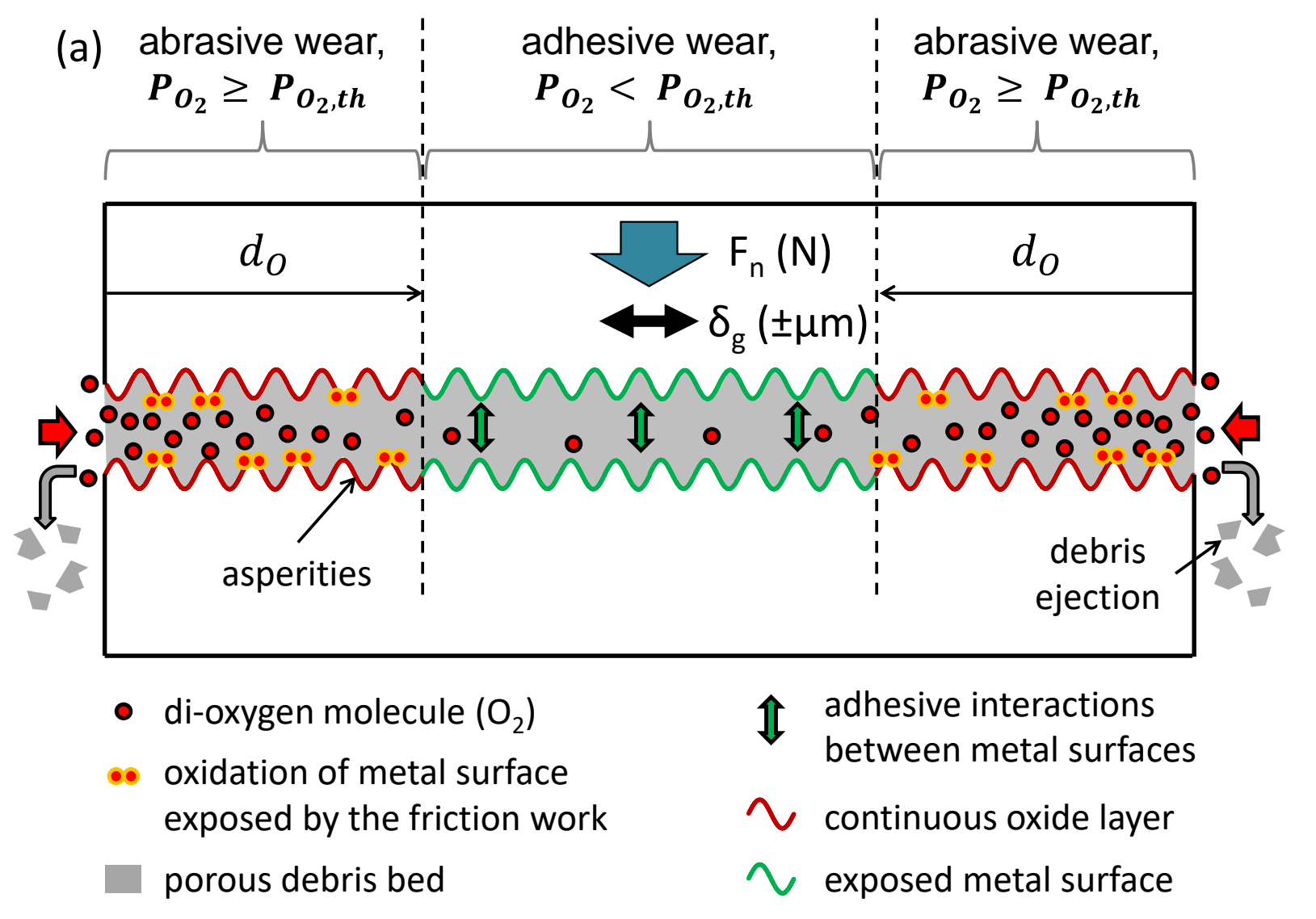

(b)

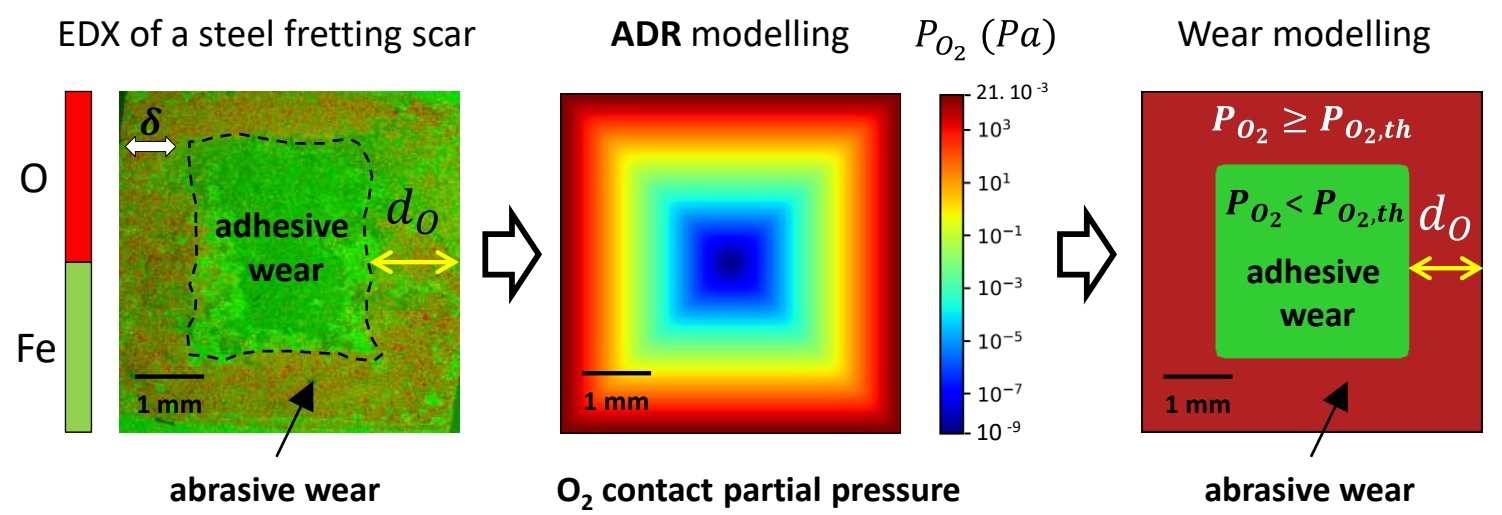

Fig. 1. (a) Schematic illustration of the oxygen transport within the interface according to the contact oxygenation concept (COC); (b) description of the advection-dispersionreaction (ADR) approach used in simulating the partition between adhesive and abrasive wear within a fretting contact [14]. 
Experimental investigations revealed that the sliding speed " $\mathrm{v}$ " and the contact pressure "p", enclosed under the so-called "p.v" or Archard power density factor, have a substantial impact on the extension of adhesive wear in fretting contacts $[11,15,16]$. Indeed, higher "p.v" factor induce higher friction power dissipation at the interface [17]. This raises surface shear strain and the reaction rate with the interfacial dioxygen molecules causing an oxygen-starved environment towards the contact center. On the other hand, when lower sliding frequency and contact pressure are applied, friction power dissipated at the interface will be lower which reduces the consumption of the dioxygen molecules towards the center shifting the contact toward full abrasive-oxidational wear.

$\mathrm{COC}$ is recently simulated by modelling oxygen transport within fretting interface using an advection-dispersion-reaction approach (ADR) by considering debris bed as a compact porous medium traversed by atmospheric gases (Fig. 1a \& b) [14]. Results showed that ADR approach can predict the partition of abrasion and adhesion as well as the transition of wear mechanisms from pure abrasive to mixed abrasive-adhesive wear at different sliding frequencies, contact pressures and contact sizes.

However, the previous model was applied on square-shaped flat-on-flat contacts. To check the validity of this approach for other contact geometries, this work aims at extending this ADR approach to axisymmetric contacts such as circular flat-on-flat. Another objective is to find a simplified analytical solution for the ADR equation so that the influence of debris bed properties can be clearly understood. 


\section{ADR axisymmetric model description}

Advection-dispersion-reaction ADR approach, which was fully detailed elsewhere [14], will be briefly reminded in this section and summarized in Fig. 2. In this model, debris bed is considered as a compact dry powdery porous medium permitting the transport of atmospheric gases namely oxygen and nitrogen.

The continuity equation of the advection-dispersion-reaction (ADR) process of a gas constituent " $i$ " in cylindrical coordinates is given by:

$$
\begin{aligned}
& a \frac{d P_{i}}{d t}=-\nabla \cdot\left(J_{i}\right)+R_{i}=-\nabla \cdot\left(J_{a, i}+J_{d, i}\right)+R_{i}-\nabla \cdot\left(-D_{i} \nabla P_{i}+v P_{i}\right)+R_{i} \\
& a \frac{d P_{i}}{d t}=-\nabla \cdot\left(J_{i}\right)+R_{i}=-\frac{1}{r} \frac{\partial\left(r J_{i(r)}\right)}{\partial r}-\frac{1}{r} \frac{\partial J_{i(\theta)}}{\partial \theta}-\frac{\partial J_{i(z)}}{\partial z}+R_{i}
\end{aligned}
$$

Such that, for a gas component " $i$ ", $P_{i}$ is the gas partial pressure, $R_{i}$ the reaction rate, $J_{i}$ the general molar flux, $J_{a, i}$ the advective flux, $J_{d, i}$ the dispersive flux, $D_{i}$ the dispersion coefficient, $a$ the porosity, $v$ the advection velocity of the gas mixture in the debris bed, $P$ the total pressure of the gas mixture, $\mu$ the viscosity of the entire gas mixture, $k$ the intrinsic permeability of the debris bed, $d_{p}$ the average particle size in the debris bed, and $r, \theta, z, \theta$ and $t$ are respectively the radial, angular, vertical and time dimensions. These physical quantities are estimated using the equations and the models shown in Fig. 2 and are fully detailed elsewhere [14].

In the given model, the reactivity with nitrogen gas is not taken into account (i.e. $R_{N_{2}} \approx 0$ ) although some nitriding phenomena are detected $[11,18]$ as revealed in [14]. On the other hand, the reaction term of the oxygen gas $\left(R_{O_{2}}\right)$ is represented as a first-order 
decay rate of oxygen gas which is consumed during oxidation reaction due to the progressive elimination of the protective oxide layer during fretting solicitations exposing in turn new fresh metal surfaces to react with the available di-oxygen molecules.

$R_{O_{2}}=-r_{O_{2}} \times C_{O_{2}}$

The oxidation rate coefficient $r_{O_{2}}$ represents the reaction rate between the exposed metal surface and the dioxygen molecules available in the interface. It is formalized using a simple phenomenological empirical expression based on the Archard power density factor $\omega$ which is equal to the so-called "p.v" factor (with "p" being the pressure and "v" being the sliding speed). Note that this formula was calibrated in a former paper [14] using only three fretting tests of $34 \mathrm{NiCrMo} 16$ flat-on-flat interface and was validated for several loading parameters.

$r_{O_{2}}=\beta\left(\frac{\omega}{\omega_{\text {ref }}}\right)^{\gamma}$ with $\omega=p \times \mathrm{v}=4 . p . \delta_{g} . f$

$\omega_{\text {ref }}$ is the Archard power density factor at the reference conditions in [14] (number of cycles $\mathrm{N}=20000$ cycles, contact pressure $p=100 \mathrm{MPa}$, sliding amplitude $\delta_{g}= \pm 100 \mu \mathrm{m}$, sliding frequency $f=1 \mathrm{~Hz}$, and contact area $A=5 \times 5=25 \mathrm{~mm}^{2}$ ) so that $\omega_{\text {ref }}=0.04 \mathrm{~W} / \mathrm{mm}^{2}$. $\beta$ and $\gamma$ are the calibration coefficients which were found to be equal to $\beta=394.63$ and $\gamma=0.47[13]$. 


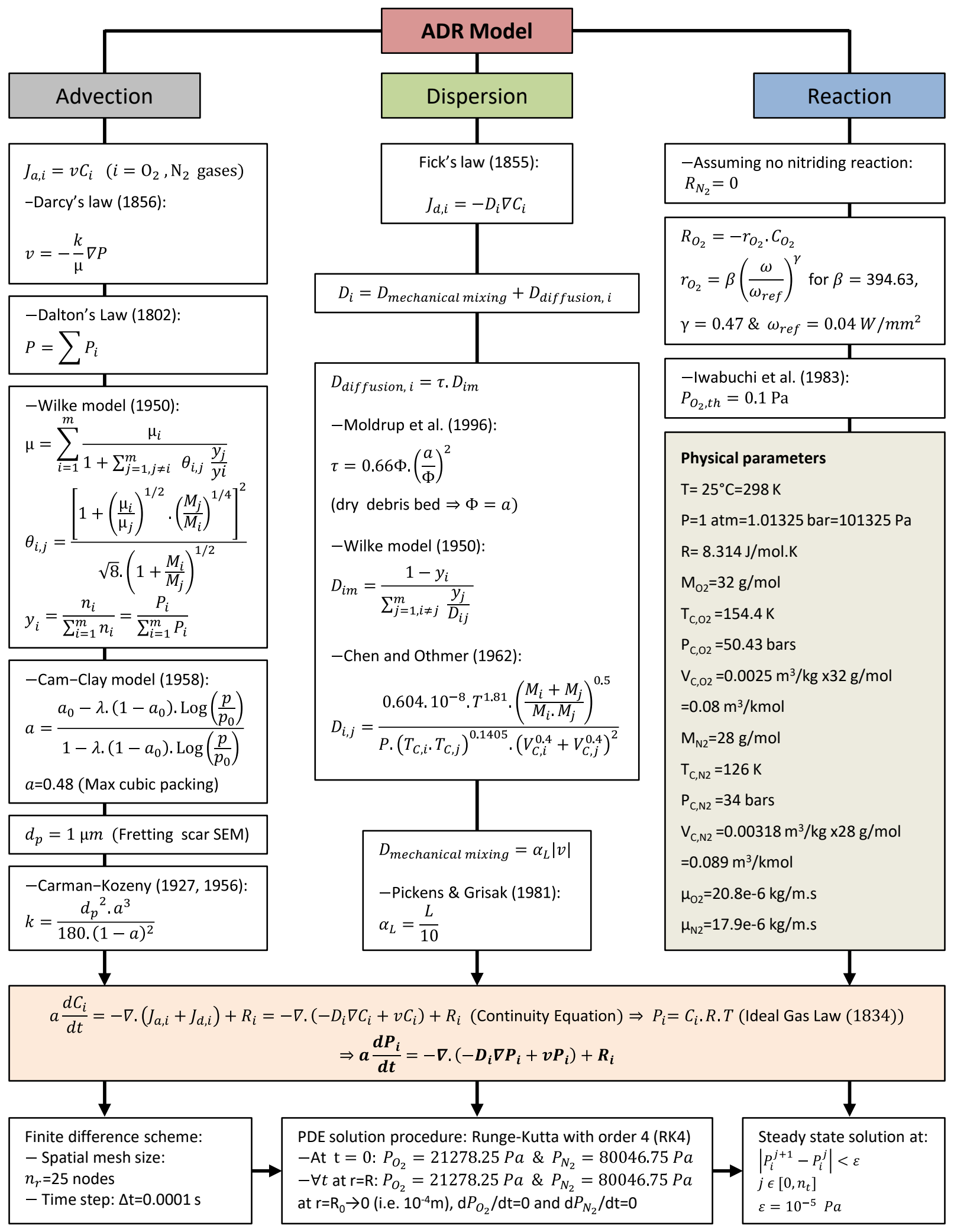

Fig. 2. Flow chart presentation of the input data, models and the numerical solution of the ADR model (after [14]). 
Assuming a homogeneous debris bed made up of identical cubically packed spherical particles, the gas flow will not vary significantly in $\theta$ and $z$ directions when compared to radial direction $r$ (Fig. 3) where there will be oxygen gas consumption towards the contact center; hence:

$$
\begin{aligned}
& \frac{\partial J_{i(\theta)}}{\partial \theta}=\frac{\partial J_{i(z)}}{\partial z}=0 \\
& a \frac{d P_{i}}{d t}=-\frac{\partial J_{i(r)}}{\partial r}-\frac{J_{i(r)}}{r}+R_{i}
\end{aligned}
$$

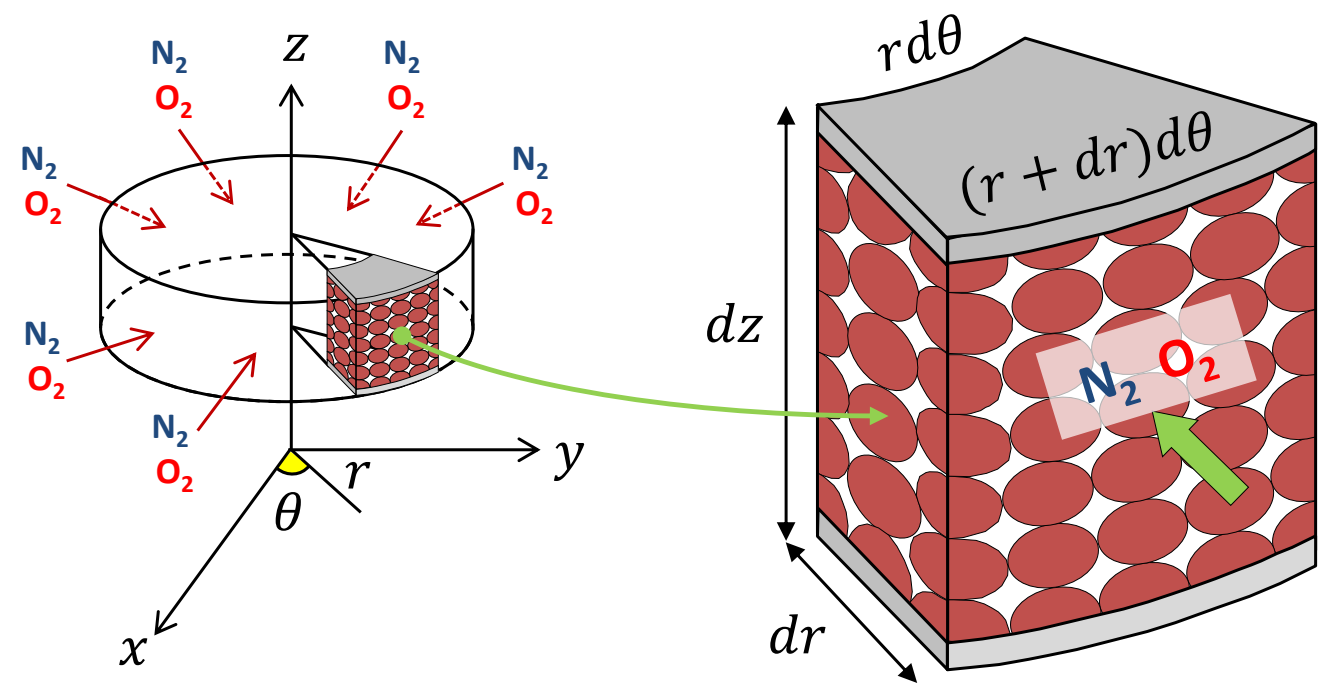

Fig.3. Schematic illustration of the radial gas transport in an axisymmetric interface.

\section{Numerical solution of the ADR continuity equation}

The continuity equation will be solved numerically by applying a finite difference scheme which was previously described by Stein and co-authors [19]. The contact radius $(R)$ is discretized into " $p$ " segments centered at nodes $\left(n_{p}\right)$ where gas partial pressures vary linearly between these nodes. Following this, a central difference approximation is 
applied for the both first and second order space derivatives of the general flux yielding the continuity equation below:

$a_{(p)} \frac{d P_{i(p)}}{d t}=\frac{J_{i\left(p-\frac{1}{2}\right)}-J_{i\left(p+\frac{1}{2}\right)}}{\Delta r}+\frac{J_{i(p)}}{r}+R_{i}$

The discretization of the time domain is expressed as follows by applying a forward difference approximation:

$\frac{P_{i(p)}^{j+1}-P_{i(p)}^{j}}{\Delta t}=\frac{J_{i\left(p-\frac{1}{2}\right)}-J_{i\left(p+\frac{1}{2}\right)}}{\Delta r \cdot a_{(p)}}-\frac{J_{i(p)}}{r \cdot a_{(p)}}+\frac{R_{i}}{a_{(p)}}$

$P_{i(p)}^{j+1}=P_{i(p)}^{j}+\Delta t \cdot\left(\frac{J_{i\left(p-\frac{1}{2}\right)}-J_{i\left(p+\frac{1}{2}\right)}}{\Delta r \cdot a_{(p)}}-\frac{J_{i(p)}}{r \cdot a_{(p)}}+\frac{R_{i}}{a_{(p)}}\right)$

$P_{i(p)}^{j+1}=P_{i(p)}^{j}+\Delta t . f_{i(p)}^{j}$

for $i \epsilon[1, m], p \in\left[1, n_{r}\right], j \in\left[0, n_{t}\right]$

The general flux of a gas component " $i$ " is discretized through the lower boundary (Eq. 9), the upper boundary (Eq. 10) and finally the lower and upper boundaries (Eq. 11) of segment $p$ such that:

$J_{i\left(p-\frac{1}{2}\right)}=-\left[D_{i\left(p-\frac{1}{2}\right)} \cdot \frac{P_{i(p)}-P_{i(p-1)}}{\Delta r}\right]-\left[\frac{k\left(p-\frac{1}{2}\right)}{\mu_{\left(p-\frac{1}{2}\right)}} \cdot \frac{P_{(p)}-P_{(p-1)}}{\Delta r} \cdot \frac{P_{i(p)}+P_{i(p-1)}}{2}\right]$
$J_{i\left(p+\frac{1}{2}\right)}=-\left[D_{i\left(p+\frac{1}{2}\right)} \cdot \frac{P_{i(p+1)}-P_{i(p)}}{\Delta r}\right]-\left[\frac{k\left(p+\frac{1}{2}\right)}{\mu} \cdot \frac{P_{(p+1)}-P_{(p)}}{\Delta r} \cdot \frac{P_{i(p+1)}+P_{i(p)}}{2}\right]$
$J_{i(p)}=-\left[D_{i(p)} \cdot \frac{P_{i(p+1)}-P_{i(p-1)}}{2 \cdot \Delta r}\right]-\left[\frac{k_{(p)}}{\mu_{(p)}} \cdot \frac{P_{(p+1)}-P_{(p-1)}}{2 \cdot \Delta r} \cdot \frac{P_{i(p+1)}+P_{i(p-1)}}{2}\right]$

Runge-Kutta method with order 4 (RK4) is applied to solve the partial differential equation of the ADR model as detailed in [14]. This method allows having a precise 
solution with relatively small computation costs. A stopping criteria is then defined to obtain the steady-state solution whereby the latter is reached if the difference between the pressure profiles between two consecutive instants of time is lower than a certain predefined value " $\varepsilon$ " such that:

$\left|P_{i}^{j+1}-P_{i}^{j}\right|<\varepsilon \Rightarrow$ steady state is reached

\section{Comparison between axisymmetric and Cartesian ADR models}

To check the validity of the ADR model in axisymmetric coordinates, the latter is compared to Cartesian ADR model at the reference conditions $(\mathrm{N}=20000$ cycles, $\mathrm{p}=100$ MPa, $\delta_{g}= \pm 100 \mu \mathrm{m}$, and $\mathrm{f}=1 \mathrm{~Hz}$ ). Fig. 4 pictures a very nice compatibility in the evolution of the di-oxygen partial pressure with respect to the radial distance. This leads to a difference in oxygen distance which is less than $5 \%$. 
(a)

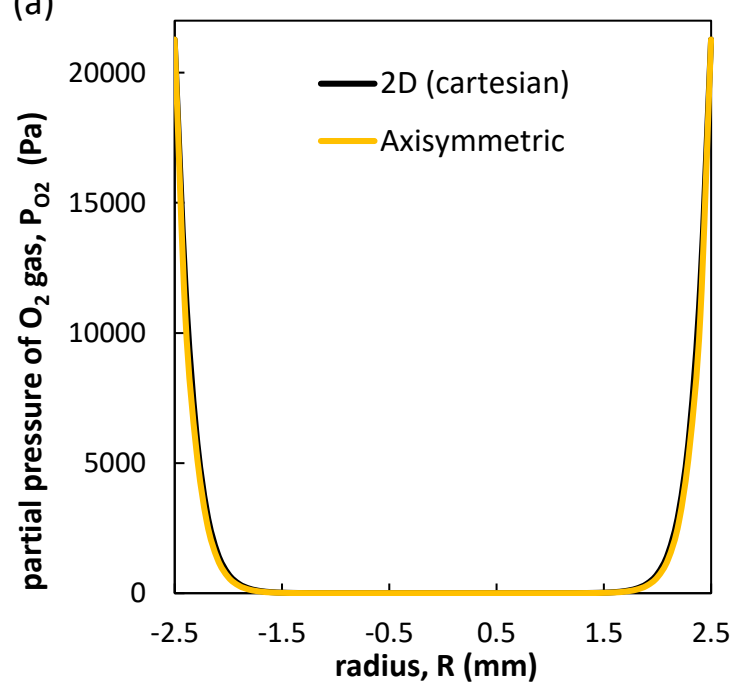

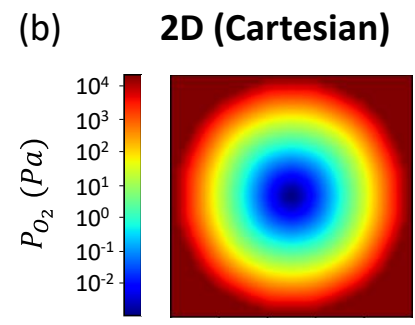

Axisymmetric

$\mathrm{O}_{2}$ partial pressure, $P_{\mathrm{O}_{2}}(\mathrm{~Pa})$

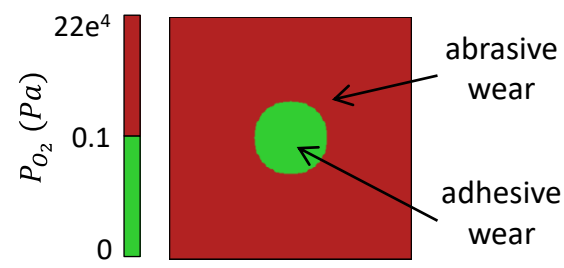

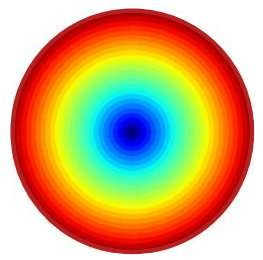

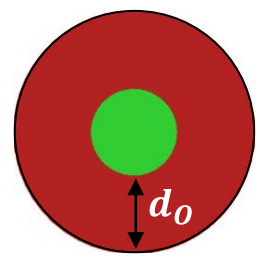

Fig. 4. Comparison between axisymmetric and Cartesian ADR approaches: (a) evolution of the di-oxygen partial pressure with respect to the radial distance; (b) 2D maps showing the evolution of the di-oxygen partial pressure at the reference conditions $\left(\mathrm{N}=20000\right.$ cycles, $\mathrm{p}=100 \mathrm{MPa}, \delta_{\mathrm{g}}= \pm 100 \mu \mathrm{m}$, and $\mathrm{f}=1 \mathrm{~Hz}$ for $\mathrm{R}=2.5 \mathrm{~mm}$ ).

\section{Experimental procedure}

\subsection{Material}

To validate this axisymmetric ADR approach, a homogeneous 34NiCrMo16 interface is used such that the reaction rate calibration coefficients were found using the same low alloyed steel in a former study in [14]. The mechanical properties and the chemical composition of this alloy are shown in Table 1. 
Table 1. Mechanical properties and chemical composition of 34NiCrMo16.

\begin{tabular}{|c|c|c|c|c|c|c|c|c|c|}
\hline \multicolumn{7}{|c|}{ Mechanical properties of 34NiCrMo16 (documentation of material supplier [20]) } \\
\hline Young's modulus, E (GPa) & Poisson's ratio, v & \multicolumn{2}{c|}{ Yield stress (0.2\%), $\sigma_{\mathrm{y} 0.2 \%}(\mathrm{MPa})$} & \multicolumn{2}{c|}{ Ultimate stress, $\sigma_{\mathrm{u}}(\mathrm{MPa})$} \\
\hline \multicolumn{2}{|c|}{0.3} & \multicolumn{3}{|c|}{950} & \multicolumn{3}{c|}{1130} \\
\hline \multicolumn{7}{|c|}{ Chemical composition of 34NiCrMo16 [21] } \\
\hline Element & $\mathrm{C}$ & $\mathrm{Si}$ & $\mathrm{Mn}$ & $\mathrm{P}$ & $\mathrm{S}$ & $\mathrm{Cr}$ & $\mathrm{Mo}$ & $\mathrm{Ni}$ & $\mathrm{Fe}$ \\
\hline \% Weight & 0.37 & 0.38 & 0.39 & 0.012 & 0.003 & 1.72 & 0.3 & 3.83 & 93 \\
\hline
\end{tabular}

\subsection{Contact configuration and experimental set-up}

A particular flat-on-flat contact geometry is used in this study consisting of a circular bottom sample (pin) of radius $\mathrm{R}=2.5 \mathrm{~mm}$ tested against flat upper specimen (plane) (Fig. 5a). The objective behind this contact configuration is to test the ADR approach in axisymmetric interface while maintaining a constant contact area and consequently constant contact pressure during wear extension.
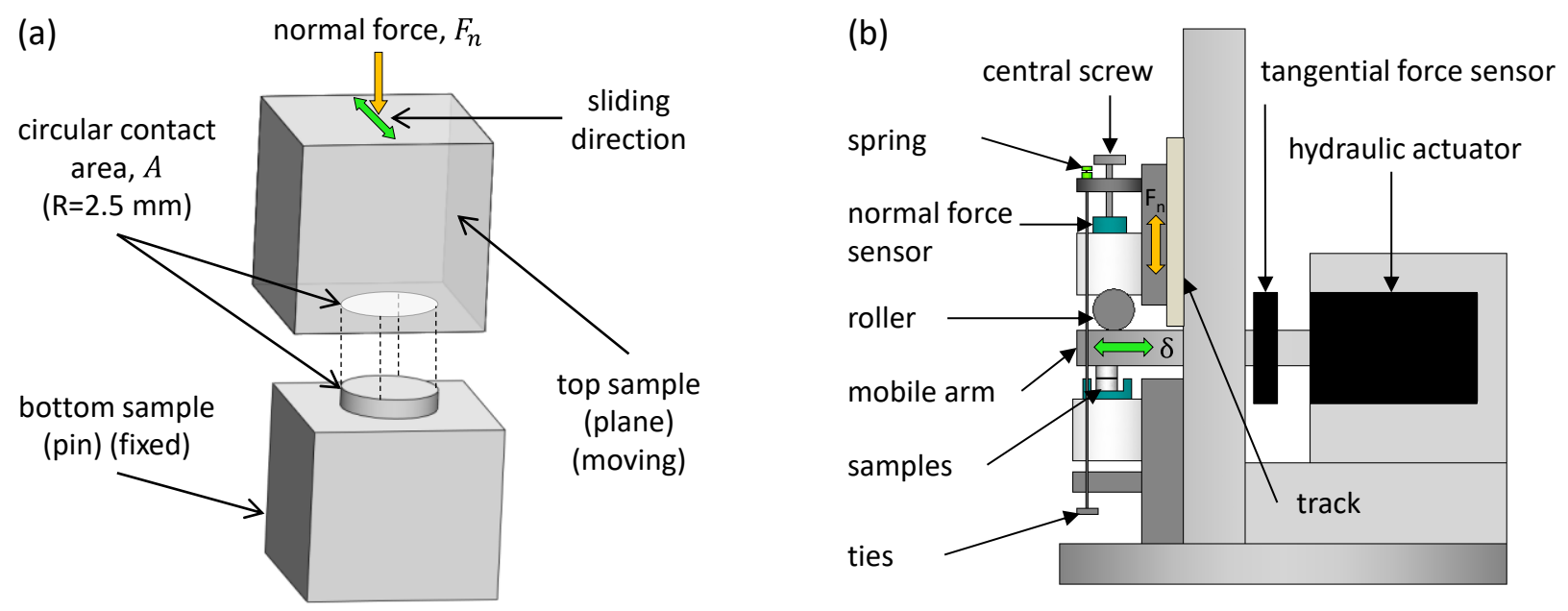

Fig. 5. Schematic illustration of the: (a) circular axisymmetric flat-on-flat contact geometry; (b) fretting wear test rig. 
Fretting wear experiments were carried out using a hydraulic test rig (Fig. 5b) which is specifically customized for examining large horizontal flat-on-flat contacts. A full description of the experimental set-up is given in $[12,13]$.

\subsection{Experimental conditions}

Fig. 6 illustrates the cross-test strategy applied to validate the ADR approach on axisymmetric contacts. Two sets of tests were performed starting from a reference experiment having $\left(\mathrm{N}=20000\right.$ cycles, $\mathrm{p}=100 \mathrm{MPa}, \delta_{g}= \pm 100 \mu \mathrm{m}$, and $\mathrm{f}=1 \mathrm{~Hz}$ for $\mathrm{R}=2.5$ $\left.\mathrm{mm}, A=19.64 \mathrm{~mm}^{2}\right)$. The first one consists in varying the sliding frequency from $\mathrm{f}=0.5$ to $10 \mathrm{~Hz}$ while keeping the other parameters constant at the reference values. In the second set, the contact pressure is varied from $p=25$ to $175 \mathrm{MPa}$ while maintaining the other parameters at the reference conditions. Fretting experiments were carried out at ambient temperature $\left(25^{\circ} \mathrm{C} \pm 5^{\circ} \mathrm{C}\right)$ and relative humidity $(\mathrm{RH}=40 \% \pm 10 \%)$.

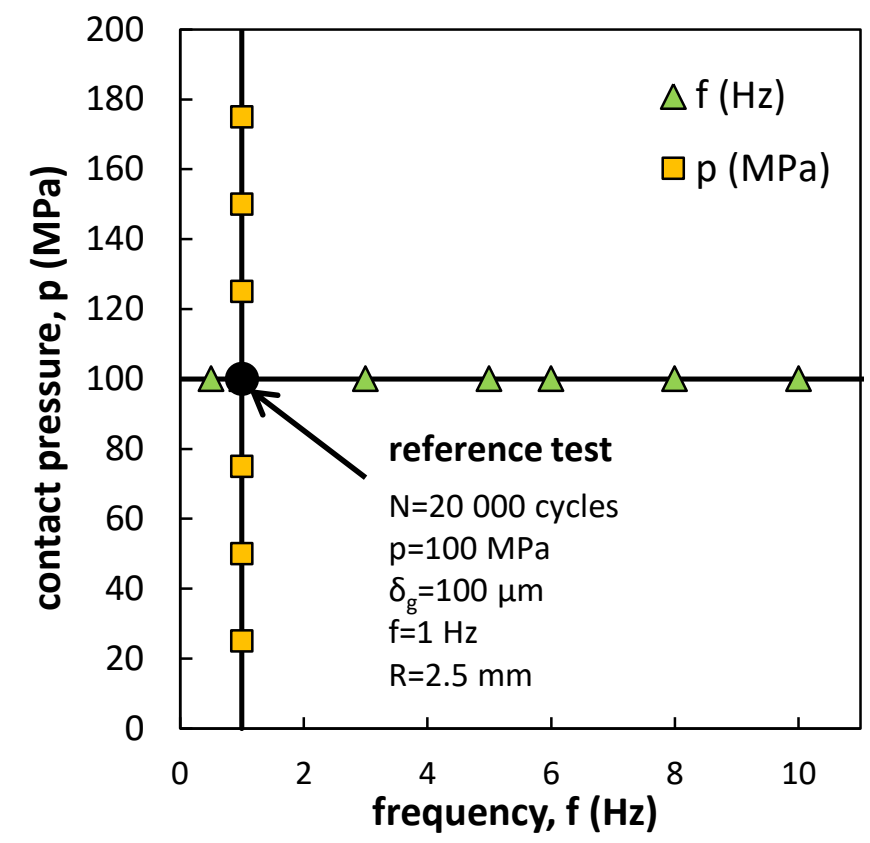

Fig. 6. Experimental map used to validate the axisymmetric wear model. 
5.4 Computation of oxygen distance and adhesive wear extension

After each test, a considerable quantity of third body is ejected as can be seen in Fig. 7a. Following this, the top and bottom samples are cleaned using ultrasonic ethanol bath for 20 minutes to remove the adhering oxide debris. EDX maps (Fig. 7b) and crossed oxygen line scans (Fig. 7c) are then acquired which allows the computation of the oxygen distance parameter " $d_{O}$ " (Fig. 7b) (as fully detailed in [13]):

$$
d_{O}=\frac{1}{2} \cdot\left(d_{O, X}+d_{O, Y}\right)
$$

The adhesion area $A_{a d}$ can be estimated from the internal zone area where the oxygen intensity is relatively low such that:

$A_{a d}=\pi \cdot\left(R-d_{o}\right)^{2}$

The abrasion area $A_{a b}$ is inferred from the measured adhesion area $A_{a d}$ and the total apparent contact area $A$ such that:

$A_{a b}=A-A_{a d}$

The relative proportions of abrasion and adhesion areas are respectively given by:

$$
\begin{aligned}
& \% A_{a b}=\frac{A_{a b}}{A} \\
& \% A_{a d}=\frac{A_{a d}}{A}
\end{aligned}
$$

Fig. 7d shows ADR map and are numerical oxygen line scans such that the limit between the adhesion and abrasion zones is defined by considering $P_{O_{2}, t h}=0.1 \mathrm{~Pa}$. This threshold oxygen partial pressure is set based on the experimental findings of Iwabuchi and co-workers [22] who revealed that oxidative wear of an iron-based alloy is significant 
when oxygen partial pressure is above $P_{O_{2}, t h}=0.1 \mathrm{~Pa}$ and adhesive transfer of metallic debris appears below $P_{O_{2}, t h}=0.1 \mathrm{~Pa}$. Interestingly, the axisymmetric ADR map and oxygen line scans appear highly correlated with the experimental results where adhesion wear is detected in the center of the fretting scar (green=very poor oxygen concentration, $\left.P_{O_{2}}<P_{O_{2}, \text { th }}\right)$ whereas abrasive-oxidational wear is observed in the $d_{O}$ width corona (brick red=high oxygen concentration, $P_{O_{2}} \geq P_{O_{2}, t h}$ ). Another point worth mentioning is that both ADR and experimental oxygen map reveal a circular adhesive internal zone which lends support to the radial isotropic flow of oxygen towards the center of the scar.

(a) Optical images before and after cleaning
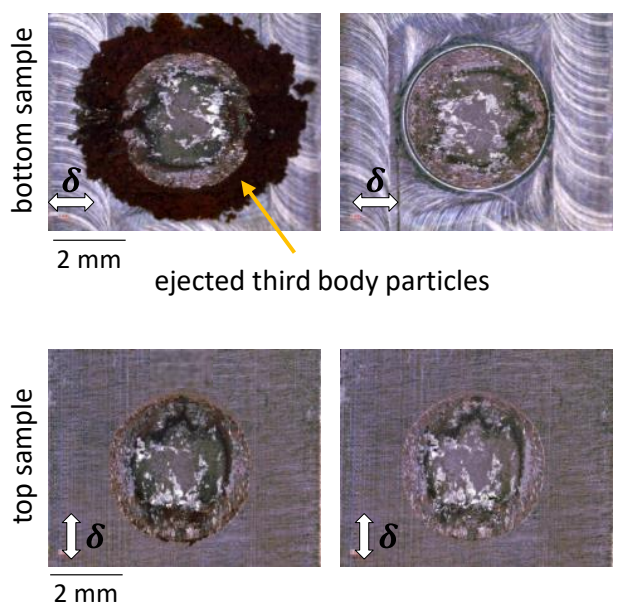

(b) EDX maps

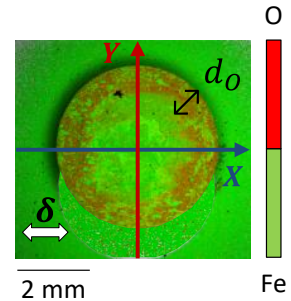

0

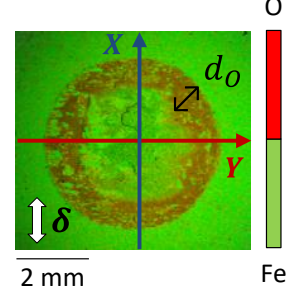

(c) EDX O-line scans
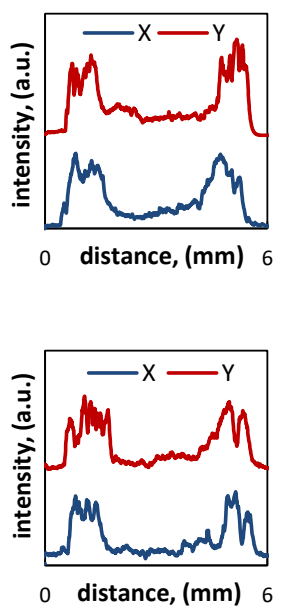

(e) ADR map \& O-line scan
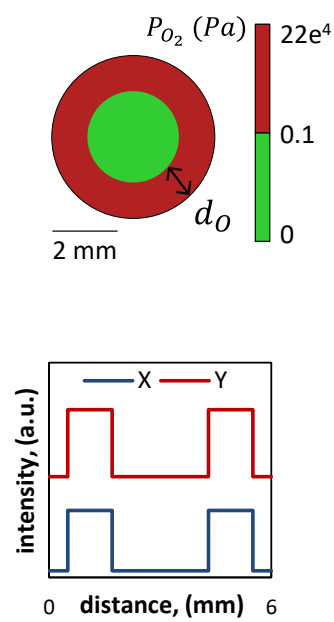

Fig. 7. (a) Optical images of the top (plane) and bottom (pin) sample before and after cleaning in ultrasonic ethanol bath; (b) EDX maps and oxygen line scans for the top and bottom sample; (c) ADR map and ADR line scan for axisymmetric contact ( $N=20000$ cycles, $p=100 \mathrm{MPa}, \delta_{g}= \pm 100 \mu \mathrm{m}, \mathrm{f}=5 \mathrm{~Hz}$ and $\mathrm{R}=2.5 \mathrm{~mm}$ ). 


\section{Results}

\subsection{Influence of the sliding frequency $(f)$}

In this section, the sliding frequency is varied from 0.5 to $10 \mathrm{~Hz}$ while keeping the other parameters constant at the reference conditions (Fig. 6). Fig. 8 reflects a very nice correlation between axisymmetric ADR maps and the experimental EDX maps and optical images. Both ADR and EDX maps reveal an extension of the adhesion zone with the sliding frequency. Additionally, a circular adhesion zone can be discerned from EDX maps and optical images especially at high sliding frequencies which once again support the hypothesis of radial flow of oxygen gas to the center of the scar.
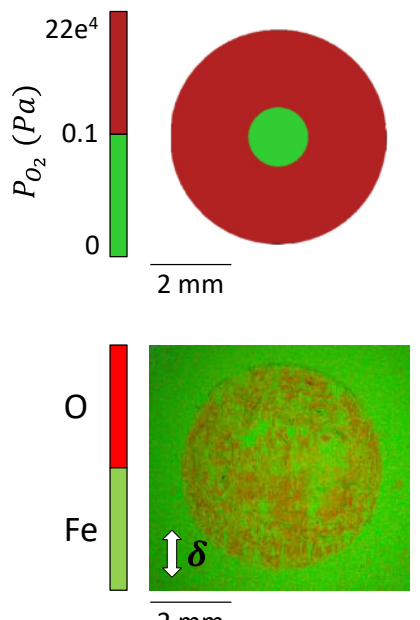

$2 \mathrm{~mm}$

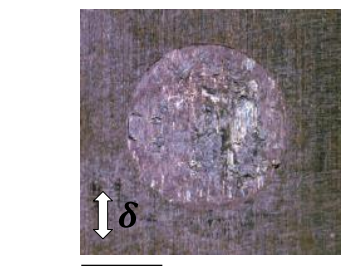

$2 \mathrm{~mm}$

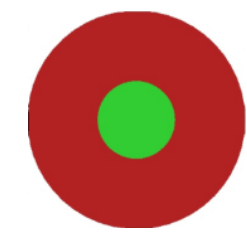

$2 \mathrm{~mm}$

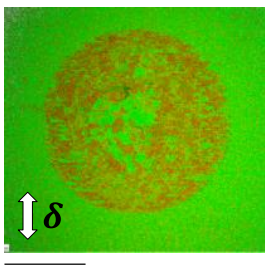

$2 \mathrm{~mm}$

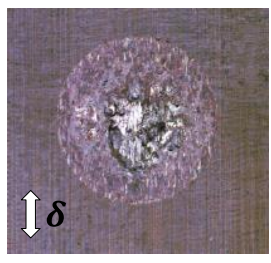

$2 \mathrm{~mm}$

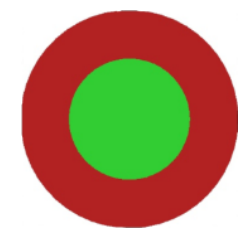

$2 \mathrm{~mm}$

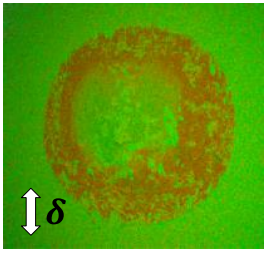

$2 \mathrm{~mm}$

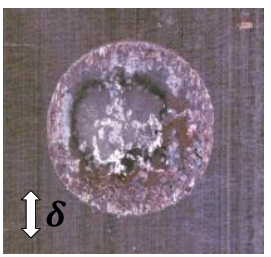

$\overline{2 \mathrm{~mm}}$

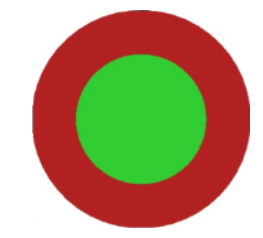

$2 \mathrm{~mm}$

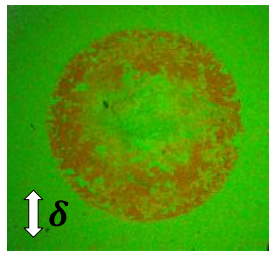

$2 \mathrm{~mm}$

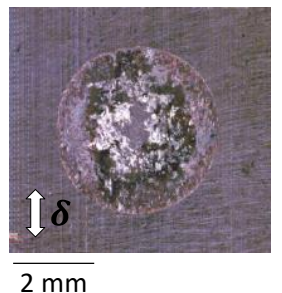

Optical images

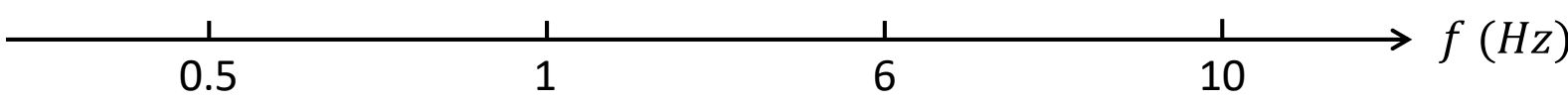

Fig. 8. Comparison between EDX and numerical ADR maps and optical images showing the expansion of adhesion zone with the increase in the sliding frequency from 0.5 to 10 $\mathrm{Hz}\left(\mathrm{N}=20000\right.$ cycles, $\mathrm{p}=100 \mathrm{MPa}, \delta_{g}= \pm 100 \mu \mathrm{m}$, and $\left.\mathrm{R}=2.5 \mathrm{~mm}\right)$. 
Fig. 9 displays the decreasing evolution of the oxygen distance " $d_{O}$ " in axisymmetric interface as a function of the sliding frequency. It appears that the axisymmetric ADR model can also quantitatively predict the recession of abrasive-oxidational wear and the associated reduction of oxygen distance as the sliding frequency increases. This tendency can be explained by the fact that increasing the sliding frequency enhances the reaction with the available dioxygen molecules which leads to their faster consumption by the exposed metal surface and consequently their deficiency from the center.

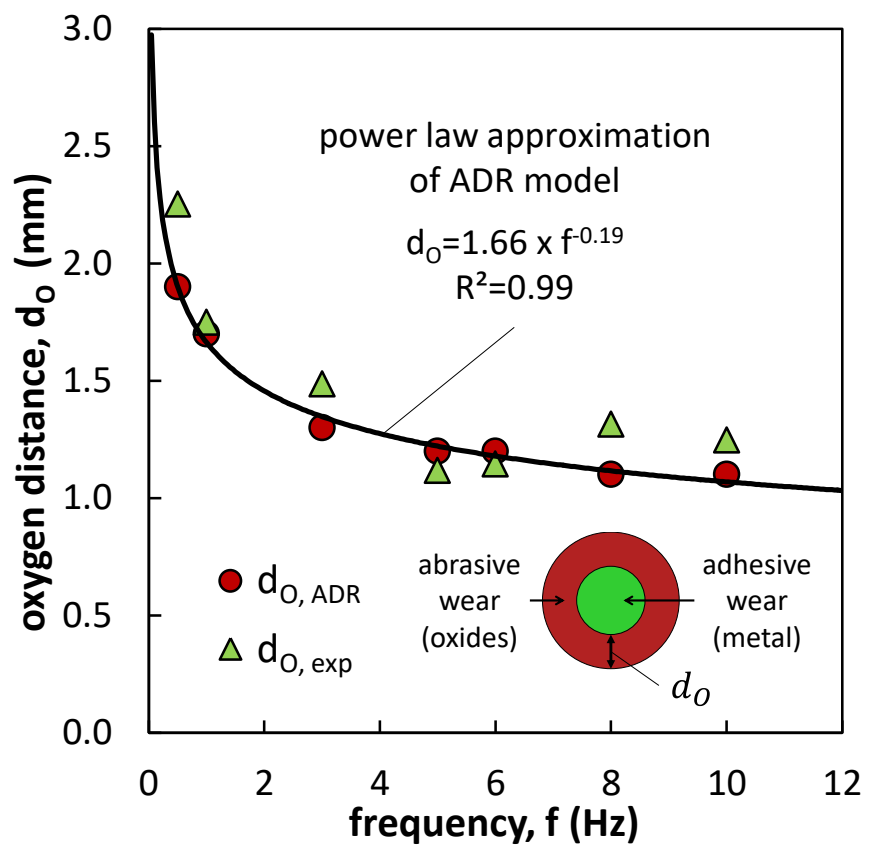

Fig. 9. Comparison between the experimental $\left(\mathrm{d}_{\mathrm{O}, \text { exp }}\right)$ and the predicted oxygen distance from the axisymmetric $A D R$ approach $\left(\mathrm{d}_{\mathrm{O}, \mathrm{ADR}}\right)$ with respect to the sliding frequency $\left(\mathrm{N}=20000\right.$ cycles, $\mathrm{p}=100 \mathrm{MPa}, \delta_{\mathrm{g}}= \pm 100 \mu \mathrm{m}$, and $\left.\mathrm{R}=2.5 \mathrm{~mm}\right)$.

6.2 Influence of the contact pressure $(p)$

Axisymmetric ADR approach is also checked at variable contact pressure as shown in Fig. 10. It can be noticed that ADR maps reflect, once again, the extension of adhesive 
wear observed in EDX maps and optical images with the increase in the contact pressure. However, it can be noticed that ADR model overestimates the adhesion area at low contact pressure and underestimates its value at high contact pressure. This comes from the fact that increasing the contact pressure not only increases the reaction rate of the fresh metal with the interfacial dioxygen gas but also decreases the porosity of the debris bed. This latter effect is not taken into account in the ADR model which assumes that the debris bed is made up of cubically packed spherical particles with constant porosity " $a=0.48$ " [14].
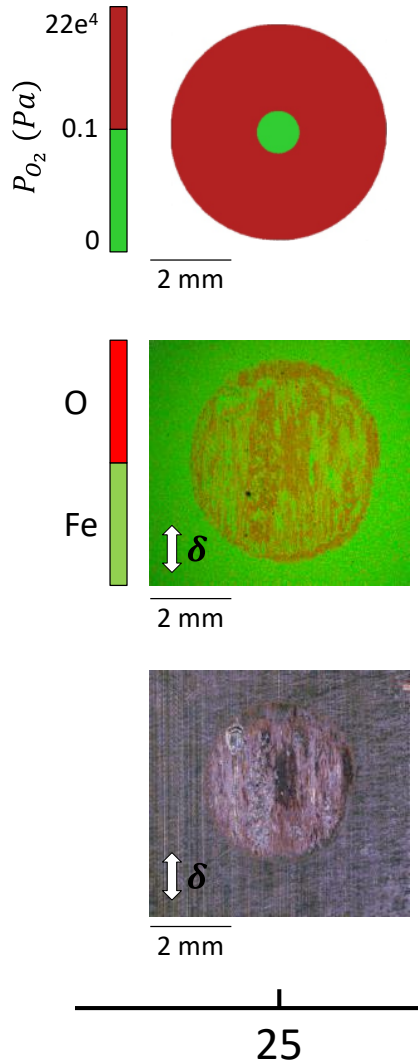
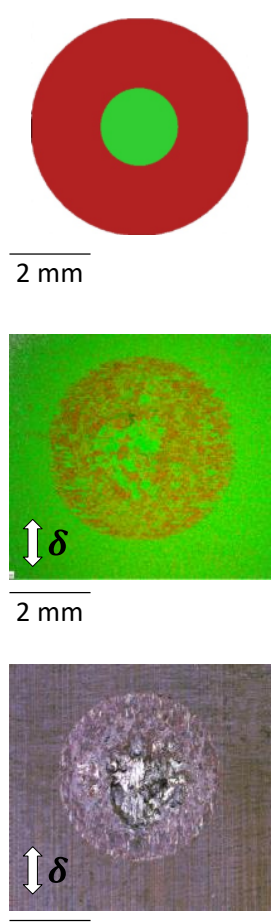

$2 \mathrm{~mm}$
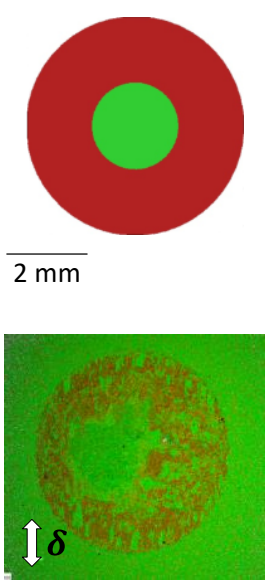

$2 \mathrm{~mm}$

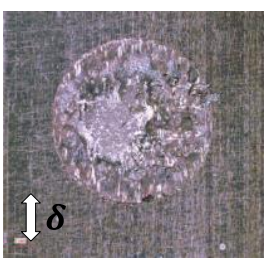

$2 \mathrm{~mm}$
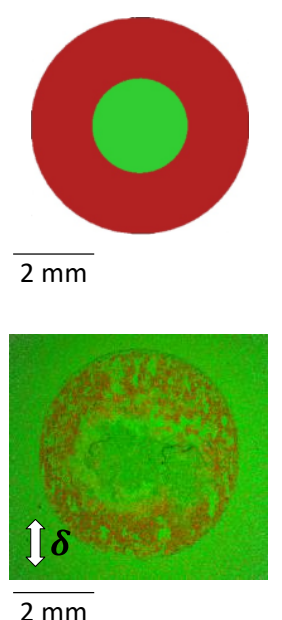

EDX maps

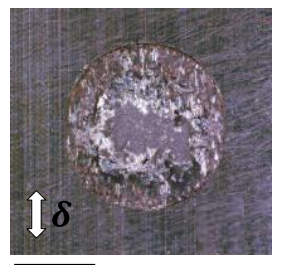

Optical images

Fig. 10. Comparison between the experimental $\left(\mathrm{d}_{0, \exp }\right)$ and the predicted oxygen distance from ADR approach $\left(\mathrm{d}_{\mathrm{O}, \mathrm{ADR}}\right)$ with respect to the sliding frequency $(\mathrm{N}=20000$ cycles, $\mathrm{p}=100 \mathrm{MPa}, \delta_{\mathrm{g}}= \pm 100 \mu \mathrm{m}$, and $\mathrm{R}=2.5 \mathrm{~mm}$ ). 
The constant-porosity effect is made clearer in Fig. 11 which shows that ADR model inherently overestimates oxygen distance at low contact pressure and underestimates the latter at high contact pressure. Nevertheless, it can still be remarked that ADR model can pertinently capture the decreasing evolution of the oxygen distance and consequently abrasion area with the contact pressure.

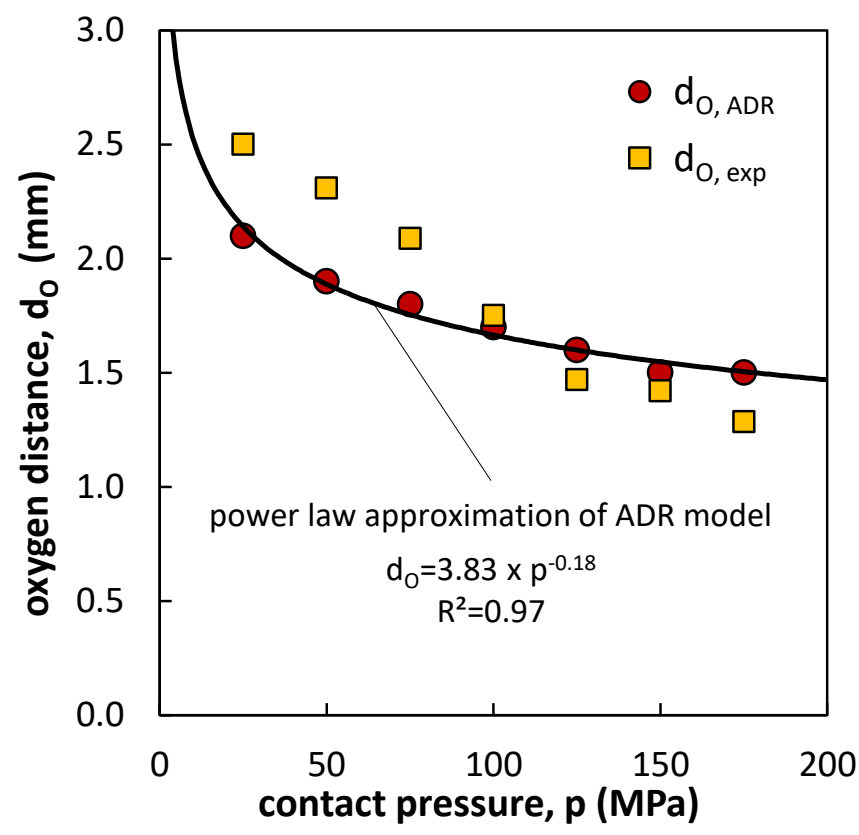

Fig. 11. Comparison between the experimental $\left(\mathrm{d}_{0, \exp }\right)$ and the predicted oxygen distance from the axisymmetric ADR approach $\left(\mathrm{d}_{\mathrm{O}, \mathrm{ADR}}\right)$ with respect to the contact pressure $\left(\mathrm{N}=20000\right.$ cycles, $\mathrm{f}=1 \mathrm{~Hz}, \delta_{g}= \pm 100 \mu \mathrm{m}$, and $\left.\mathrm{R}=2.5 \mathrm{~mm}\right)$.

\subsection{Archard power density factor formulation}

The aforementioned effects of contact pressure and siding frequency are combined in Fig. 12 which shows the variation of the experimental and numerical oxygen distance as with respect to the Archard power density factor (i.e. " $\omega=p v$ " factor). It is obvious that the numerical oxygen distance " $\mathrm{d}_{\mathrm{O}, \mathrm{ADR}}$ " dovetails neatly with the experimental one " $\mathrm{d}_{0, \exp }$ ", 
where a single master curve of oxygen distance evolution can be predicted by the axisymmetric ADR model such that:

$d_{O}=0.90 \times \omega^{-0.19}$

It should be mentioned that the above equation is very close from that obtained by the Cartesian (2D) ADR model given in [14] which indirectly supports the stability of the axisymmetric suggested approach.

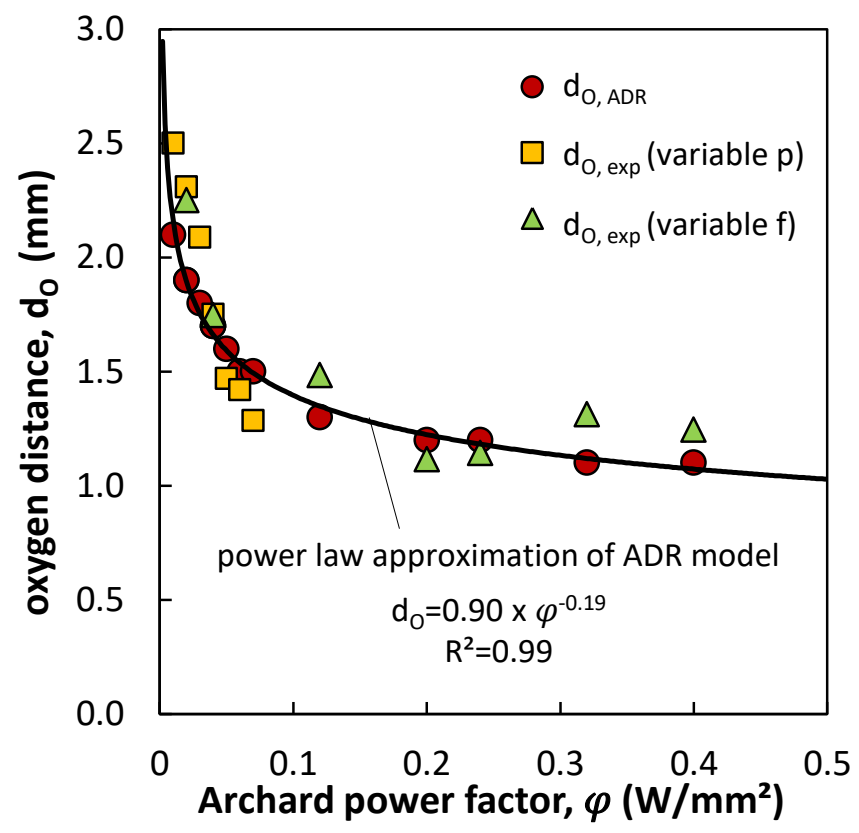

Fig. 12. Comparison between the experimental $\left(\mathrm{d}_{0, \exp }\right)$ and the predicted oxygen distance from $A D R$ approach $\left(\mathrm{d}_{\mathrm{O}, \mathrm{ADR}}\right)$ with respect to the Archard power density factor $\omega=p \times \mathrm{v}\left(\mathrm{N}=20000\right.$ cycles, $\delta_{\mathrm{g}}= \pm 100 \mu \mathrm{m}$, and $\left.\mathrm{R}=2.5 \mathrm{~mm}\right)$.

\subsection{Quantitative validation of ADR approach}

Fig. 13a, b \&c compile the correlation between experimental and axisymmetric ADR results for more than 35 tests considering variable contact pressure, sliding frequency, and contact size for both flat circular and squared shaped [14] contacts. A very nice 
correlation is reflected between the experimental and the ADR-numerical oxygen distance, abrasion and adhesion areas for both circular and square shaped configurations.
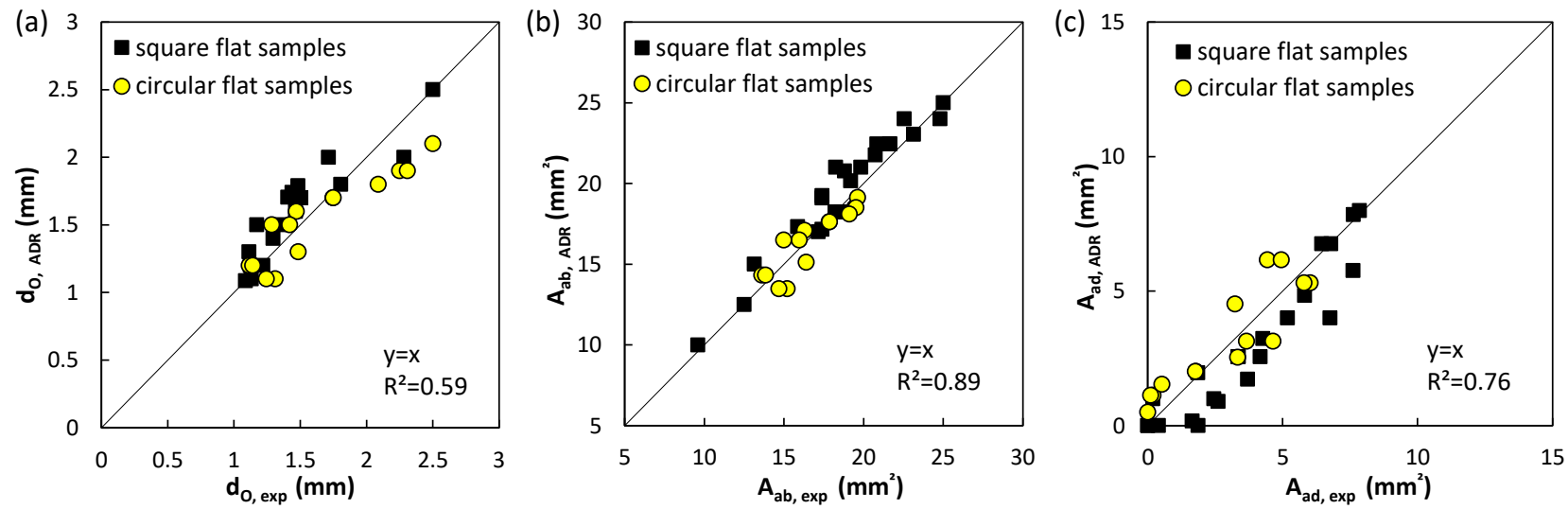

Fig. 13. Correlation between the experimental and the predicted results (axisymmetric ADR approach) for (a) the oxygen distance " $\mathrm{d}_{0}$ "; (b) the abrasion area " $\mathrm{A}_{\mathrm{ab}}$ "; and (c) the adhesion area " $\mathrm{A}_{\mathrm{ad}}$ ".

\section{Discussion}

The objective of this paper is to validate ADR model, previously examined in squareshaped interface [14], using an axisymmetric circular flat-on-flat contact. Hence, ADR model is expressed in cylindrical coordinates where the interfacial gaseous transport was assumed to be significant only in the radial direction. Fretting wear results of a flat sample sliding against a circular flat (pin) sample reveals that adhesion area extends with the augmentation of the contact pressure and sliding frequency or equivalently with the Archard power density factor $\omega=p \times \mathrm{v}$. This increase can be clarified by the contact oxygenation concept $(\mathrm{COC})[8,11,13,14]$ which suggests that dissipating more friction energy in the interface (through increasing the sliding frequency and/or the contact 
pressure) increases the rate of the oxidation reaction between the new exposed fresh metal with the interfacial oxygen gas. This leads to higher consumption of the di-oxygen molecules from the contact borders towards the center causing depletion of oxygen gas towards the center. This in turn favors abrasive-oxidational wear at the contact borders where the oxygen partial pressure is sufficiently high $\left(P_{O_{2}} \geq P_{O_{2}, t h}\right)$. On the other hand, adhesive wear prevails at the contact center where the dioxygen flow is restricted $\left(P_{O_{2}}<P_{O_{2}, t h}\right)$. What seems quite interesting in this axisymmetric ADR approach is that it can deftly mimic the circular shape of adhesion area detected experimentally which supports the hypothesis of the radial isotropic flow of oxygen gas within the interface.

The numerical axisymmetric model seems to be a relevant approach for predicting adhesive wear extension in axisymmetric contacts. However, the numerical solution of the ADR model (Eq. 1) does not reflect explicitly the influence of the material reactivity and the debris bed properties (porosity, permeability, and particle size) on the adhesive wear extension. This necessitates finding a simplified analytical solution of the ADR model (Eq. 1) which allows assessing the role of the aforesaid parameters separately. However, the presence of highly coupled parameters in ADR continuity equation (Eq. 1 \& Fig. 2) represents an important obstacle to finding an exact analytical solution. Hence, to tackle this problem, the following hypotheses are proposed to extract a simplified axisymmetric ADR continuity equation (Eq. 1):

- The steady state solution is considered such that:

$$
\frac{d P_{O_{2}}}{d t}=0
$$


- The gas flow does not vary significantly in $\theta$ and $z$ directions when compared to radial direction $r$, hence:

$\frac{\partial J_{O_{2}}(\theta)}{\partial \theta}=\frac{\partial J_{O_{2}(z)}}{\partial z}=0$

$-\frac{\partial J_{O_{2}(r)}}{\partial r}-\frac{J_{O_{2}}(r)}{r}+R_{O_{2}}=0$

- The advection, diffusion and reaction parameters are supposed to be constant in the radial direction (i.e. $D_{O_{2}}=$ constant, $v=$ constant, $r_{O_{2}}=$ constant), hence:

$D_{O_{2}} \frac{d^{2} P_{O_{2}}}{d r^{2}}+\left(\frac{D_{O_{2}}}{r}-v\right) \frac{d P_{O_{2}}}{d r}-\left(r_{O_{2}}+\frac{v}{r}\right) P_{O_{2}}=0$

This ordinary differential equation (ODE) is still complicated to solve analytically. To simplify the analytical solution of the model, the relative importance of advection to diffusion processes needs to be assessed. Indeed, when the advection velocity " $v$ " is low, gas transport will be controlled by diffusion. On the other hand, when the advection velocity is high, gas flow will be governed by advection process. In low permeability medium, gas advection usually plays a minor role in the transport phenomenon which is mostly controlled by diffusion. This was also noticed in the ADR approach in [14] where sensitivity analysis revealed that debris particle size and permeability had very effect on the results suggesting that gas transport is mostly governed by diffusion process. Hence, it is worth to check the relative significance of advection process especially in low permeability medium where eliminating advection can significantly simplify the transport equation by eliminating the nonlinearities associated with the advection and mechanical mixing terms (Fig. 2) [23]. One criterion to check this aspect is the Peclet number " $P e$ " which represents the ratio of the time required for the gas particles to travel 
a characteristic length by advection and the time needed for the particles to traverse the same length by diffusion [24] (Eq. 25). The characteristic length is the length over which the particles can diffuse which is equal to the radius of the interface and $D_{\text {diffusion, } O_{2}}$ is the effective diffusion coefficient of $\mathrm{O}_{2}$ gas [23]. Following this definition, transport will be diffusion controlled whenever $P e<1$ [23].

$P e=\frac{|v| R}{D_{\text {diffusion, } o_{2}}}$

Fig. 14 shows the evolution of the Peclet number with respect to the radial distance at the reference conditions. Since the advection velocity " $v$ " is not constant, an average value of Peclet number is estimated which is found to be equal to smaller than 1 . Hence, by assuming this average value, it can be supposed that oxygen transport in the contact is mainly driven by diffusion. Based on this finding, the transport of nitrogen gas which is mainly controlled by the advection process [14] will be considered constant yielding an interfacial nitrogen partial pressure equivalent to its value in the ambient air. By supposing that the advection is negligible with respect to diffusion and reaction processes in oxygen gas (i.e. $v=0$ ), Eq. 24 can be reduced to:

$r^{2} \frac{d^{2} P_{O_{2}}}{d r^{2}}+r \frac{d P_{O_{2}}}{d r}-(\eta r)^{2} P_{O_{2}}=0$ where $\eta=\sqrt{\frac{r_{O_{2}}}{D_{\text {diffusion, } O_{2}}}}=\sqrt{\frac{\beta\left(\frac{\omega}{\omega_{\text {ref }}}\right)^{\gamma}}{0.66 \times a \times D_{O_{2}}}}$

This equation can be solved assuming a mixed boundary condition such that:

$$
\begin{aligned}
& P_{O_{2}}(r=R=2.5 \mathrm{~mm})=21278.25 \mathrm{~Pa} \text { (Dirichlet boundary condition) } \\
& \frac{d P_{O_{2}}}{d r}\left(r=R_{0} \sim 0\right)=0 \text { (Neumann boundary condition) }
\end{aligned}
$$




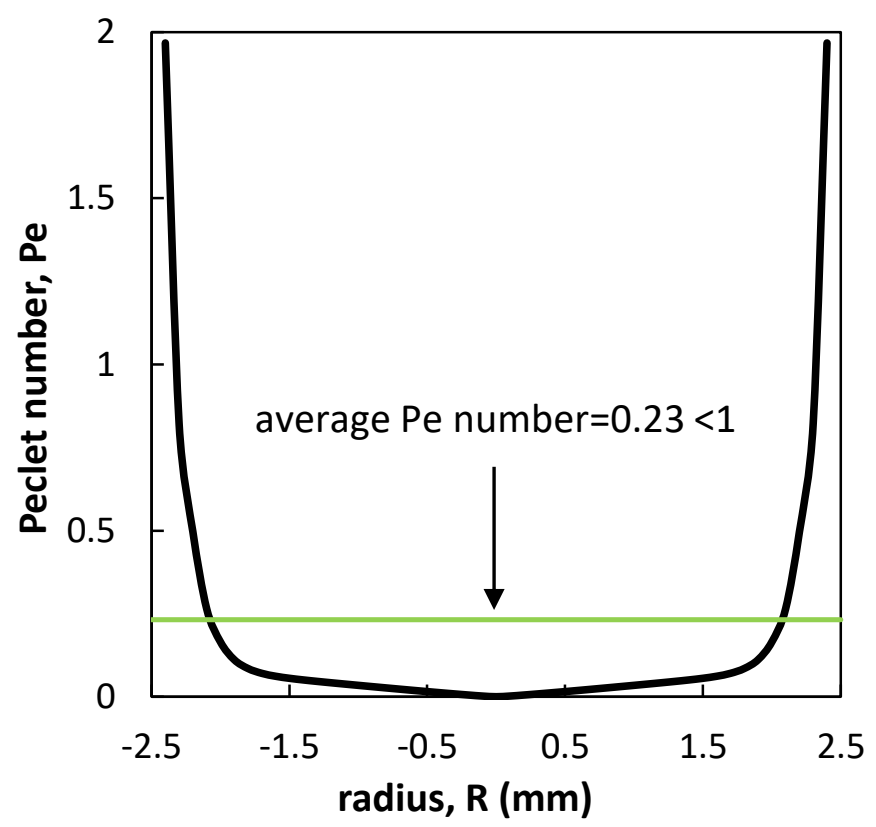

Fig. 14. Evolution of the Peclet number with the radial distance at the reference conditions $\left(\mathrm{p}=100 \mathrm{MPa}, \mathrm{f}=1 \mathrm{~Hz}, \delta_{\mathrm{g}}= \pm 100 \mu \mathrm{m}\right.$, and $\left.\mathrm{R}=2.5 \mathrm{~mm}\right)$.

Eq. 26 is a Bessel ODE of order zero whose solution is expressed as:

$P_{O_{2}}(r)=A \times I_{0}(\eta r)+B \times K_{0}(\eta r)$

$I_{0}(\eta r)=\sum_{n=0}^{\infty} \frac{1}{(n !)^{2}}\left(\frac{1}{2} \eta r\right)^{2 n}$

$K_{0}(\eta r)=\int_{0}^{\infty} \cos (\eta r \sinh (t) d t)=\int_{0}^{\infty} \frac{1}{\sqrt{t^{2}+1}} \cos (\eta r) d t$

$A=\frac{K_{1}\left(\eta R_{0}\right)}{I_{1}\left(\eta R_{0}\right) K_{0}\left(\eta R_{0}\right)+I_{1}\left(\eta R_{1}\right) K_{1}\left(\eta R_{1}\right)}$

$B=\frac{I_{1}\left(\eta R_{0}\right)}{I_{1}\left(\eta R_{0}\right) K_{0}\left(\eta R_{0}\right)+I_{1}\left(\eta R_{1}\right) K_{1}\left(\eta R_{1}\right)}$

Note that the constants " $A$ " (Eq. 32) and " $B$ " (Eq. 33) are determined by applying the boundary conditions shown in Eq. 27 and Eq. 28. 
Fig. 15 compares the numerical and the simplified analytical solutions of the axisymmetric ADR model. It seems that the analytical solution is highly correlated with the numerical one as it leads to compatible oxygen partial pressure profiles (Fig. 15a) in addition to highly correlated oxygen distance values (Fig. 15b \&c). This suggests that the analytical solution, despite the simplifications made, is highly predictive and can be considered as a simplified way to solve ADR equations.
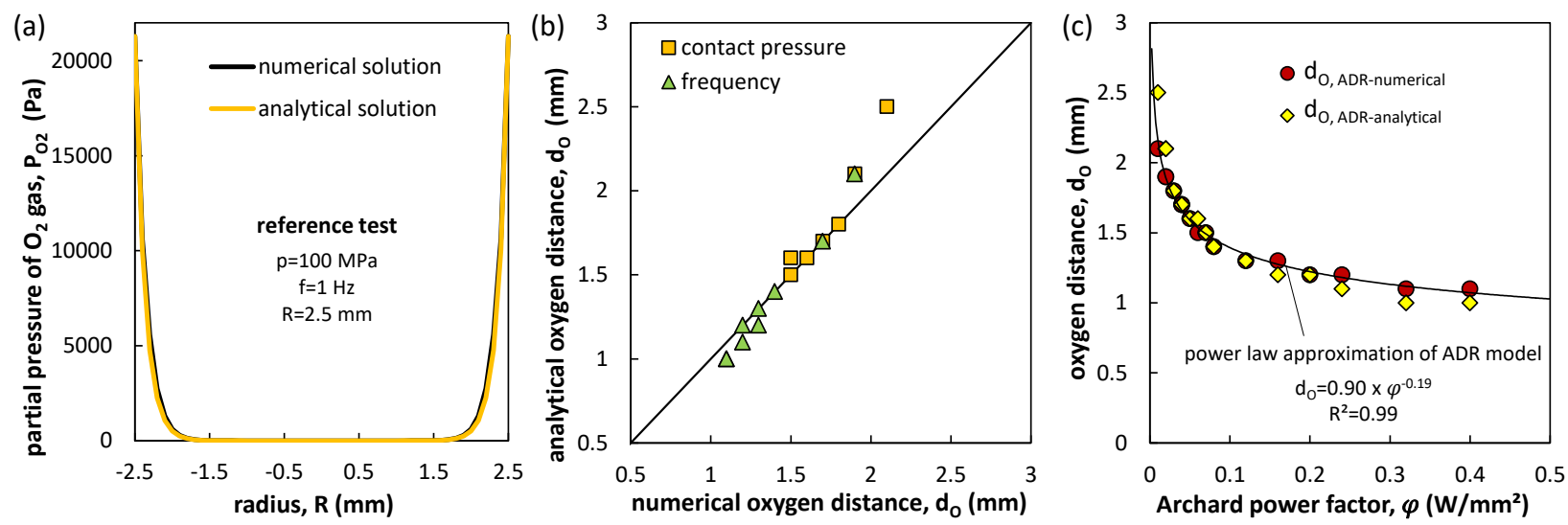

Fig. 15. (a) Comparison between the oxygen partial pressure profiles obtained by the numerical and analytical solutions of the axisymmetric ADR approach; (b) correlation between the oxygen distance values computed numerically and analytically; (c) evolution of the oxygen distance computed numerically and analytically with respect to the Archard power density factor.

One advantage of the analytical solution is that it allows studying the effect of the main parameters affecting the ADR-COC approach. Based on Eq. 28 \& 29, it appears that the reaction rate coefficient (i.e. the material reactivity under fretting solicitations triggered by the dissipated friction power $\omega=p \times \mathrm{v}$ ) and the porosity seem to be the most effective parameters in ADR approach. Fig. 16 shows the evolution of the oxygen distance values 
found by applying the simplified analytical solution versus the debris bed porosity. It appears that the porosity has a strong effect on the oxygen distance as low porosity results in very low oxygen distance and severe adhesive wear whereas high porosity shifts the contact towards a purely abrasive wear. Hence, caution should be exercised when dealing with parameter which seems indispensable for having more predictive model especially when variable contact pressure is applied. Note that this result is analogous with sensitivity analysis performed using numerical solution of the ADR approach applied on square-shaped specimens [14] where similar linear increase of oxygen distance with respect to the porosity was detected.

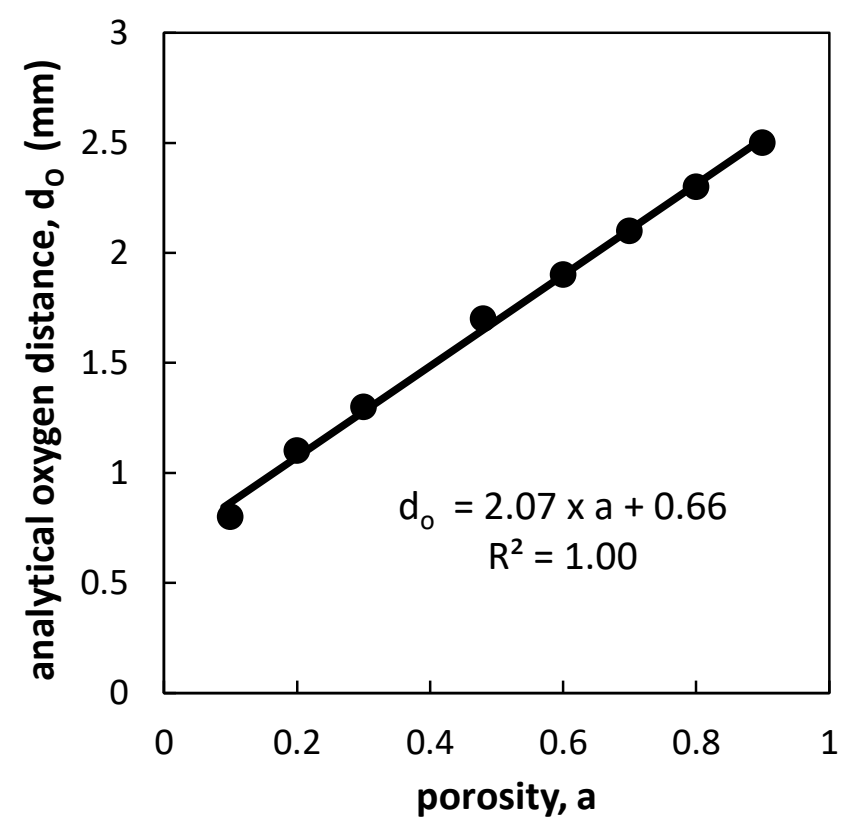

Fig. 16. Evolution of the oxygen distance determined analytically as a function of the debris bed porosity. 


\section{Conclusion}

The main target of this paper is model adhesive wear extension in axisymmetric circular contacts. To do so, contact oxygenation concept (COC) which describes the influence of fretting loadings on the dioxygen flow within the interface was modeled using an axisymmetric advection-diffusion-reaction (ADR) approach.

Axisymmetric ADR approach, which was shown to be equivalent to 2D Cartesian ADR model, was capable of predicting the circular isotropic extension of the adhesive wear at different contact pressures and sliding frequencies.

Besides, a simplified analytical solution of the axisymmetric ADR model was suggested which was highly correlated with the numerical solution and allowed an explicit investigation of the main parameters affecting the ADR-COC processes namely the porosity of the debris bed and the reaction rate coefficient which depends on the Archard power density factor. 